\title{
PROCESSO DE RECUPERAÇÃO DO TEOR METÁLICO CONTIDO NAS LAMAS DE ACIARIA*
}

\author{
Erivelto Luís de Souza ${ }^{1}$ \\ Fernando Gabriel da Silva Araújo² \\ Cristovam Paes de Oliveira ${ }^{3}$ \\ José Emanuel Lopes Gomes ${ }^{4}$
}

\begin{abstract}
Resumo
Durante o processo de fabricação do aço, em conversores LD, o ferro líquido projetado na atmosfera oxidante do conversor, solidifica-se sob a forma de pequenas esferas com uma gama variada de tamanhos. Entretanto, nem todas as esferas são perfeitas, pois algumas acabam por não conseguir completar sua esferoidização, devido às condições variadas de tamanho, velocidade de resfriamento, e tensões superficiais. Junto com as microesferas metálicas vão materiais não metálicos (escória; coque; $\mathrm{CaO}$; etc.). Após a lavagem dos gases, a "lama" formada conterá então microesferas de aço, aglomeradas entre si e com as impurezas, através da ação agregante das partículas finas, aqui denominadas de "poeira". A técnica aqui apresentada consiste na aplicação de ondas ultrassônicas sobre uma polpa. Este bombardeamento ultrassônico promove a dispersão das micropartículas ligantes da lama. Após a desagregação, as partículas que compõem a polpa estarão completamente liberadas. A polpa é então encaminhada a uma etapa de concentração gravítica para a recuperação das partículas esféricas de elevado teor metálico (90 a 96\% Fe).
\end{abstract}

Palavras-chave: Lama de Aciaria; Bombadeamento Ultrassônico; Recuperação de Resíduos.

\section{PROCESS OF RECOVERY OF THE METALLIC CONTENT CONTAINED IN STEELMAKER SLUDGE}

\section{Abstract}

During the process of manufacturing the steel, in LD converters, the liquid iron projected into the oxidizing atmosphere of the converter solidifies in the form of small spheres with a varied range of sizes. However, not all spheres are perfect, as some end up not being able to complete their spheroidization due to varying size, cooling rate, and surface stress conditions. With the metallic microspheres non-metallic materials is arrested (Slag, coke, $\mathrm{CaO}$, etc.). After washing the gases, the formed sludge will then contain steel microspheres, agglomerated with each other and with the impurities, through the aggregating action of the fine particles, herein referred to as "sludge". The technique presented here consists of the application of ultrasonic waves on a pulp. This ultrasonic bombardment promotes the dispersion of the binding microparticles. After disintegration, the particles that make up the pulp will be completely released. The pulp is then sent to a gravitational concentration step for the recovery of spherical particles of high metallic content (90 to $96 \% \mathrm{Fe}$ ).

Keywords: Steelmaker Sludge; Ultrasonic Bombardment; Waste Recover.

1 Engenheiro Metalúrgico e de Materiais, Doutor, Professor Adjunto, DTECH/CAP, UFSJ, Ouro Branco, MG, Brasil.

2 Bacharel em Física, Doutor, Professor Associado, DEFIS/UFOP, REDEMAT, Ouro Preto, MG, Brasil.

3 Engenheiro Metalúrigoc, Doutor, Presidente da Fundação Gorceix, Fundação Gorceix, Ouro Preto, MG, Brasil.

4 Engenheiro Metalúrigoc, Doutor, Presidente da Fundação Gorceix, Fundação Gorceix, Ouro Preto, MG, Brasil. 


\section{INTRODUÇÃO}

\subsection{Histórico}

Os descartes dos processos industriais são, simultaneamente, rejeito e resíduo. Nesta perspectiva, define-se como rejeito todo o material descartado antes de participar do processo, ou seja, um descarte a priori. Este descarte é feito pelo fato do processo não ter como lidar com este descarte. Ex.: a escória de alto-forno é prejudicial à aciaria, sendo assim é descartada antes do processo. Define-se então, como resíduo, o descarte a posteriori, aquele é gerado pelo próprio processo analisado. Com base nisso podemos entender que o mesmo descarte é rejeito e resíduo, dependendo de qual processo ele é avaliado.

\subsection{Problema Analisado}

A lama de aciaria se divide, por seu teor metálico, em duas partes: lama grossa $(32 \%$ da lama gerada) e lama fina ( $68 \%$ da lama gerada). O que as diferencia é seu teor metálico, e são separadas por granulometria. A lama grossa acima de $325 \#(+45 \mu \mathrm{m})$ e a lama fina abaixo desta faixa $(-45 \mu \mathrm{m})$. A lama grossa possui um teor médio de $70 \%$ de ferro metálico, na forma de microesferas de aço, formadas durante o sopro de oxigênio no conversor. $A$ lama fina apresenta uma composição media de $60 \%$ de $\mathrm{FeO}$, com um teor metálico próximo a $5 \%$. Buscou-se uma forma de retirada do teor metálico da lama grossa, bem como uma individualização das partículas das duas lamas.

\subsection{Objetivos do Processo}

O objetivo geral é dispersar as partículas que compõem a lama por meio do uso de um bombardeamento ultrassônico em equipamento desenvolvido para este fim. Os objetivos específicos foram de desagregar e dispersar as partículas da lama grossa, viabilizando a concentração do conteúdo metálico por meio de processos gravíticos operacionais, por fim, utilizar as partículas ricas em óxidos carbonatáveis para sequestro de carbono.

\section{MATERIAIS E MÉTODOS}

\subsection{Princípio Operacional do equipamento}

O ultrassom consiste em vibrações mecânicas, ou acústicas, que se propagam nos três meios conhecidos, respeitando a velocidade normal do som nestes meios, como qualquer outra onda sonora. O que diferencia o mesmo para que se destaque com relação às ondas comuns de som, consiste na freqüência de atuação do mesmo, o ultra-som se situa entre os $20 \mathrm{kHz}$ e $600 \mathrm{kHz}$, aproximadamente, ou seja, está numa faixa acústica fora do alcance humano. O equipamento projetado se baseia em princípios já estudados por VIGOUREAUX (1950).

Transdutores Piezelétricos. Um sólido é considerado piezelétrico se cargas elétricas são liberadas no mesmo quando está sujeito a tensões mecânicas. Quando o sinal da tensão é revertido, a polaridade das cargas elétricas são também revertidas. A variação da pressão piezelétrica depende linearmente da excitação do pulso elétrico.

Numa pastilha circular piezelétrica, a pressão mecânica axial pode ser expressa pela equação (1), para quando a distância $R$ não é muito maior que o raio do disco.

Onde:

$$
p=\operatorname{sv} \cdot 2 \operatorname{sen} \frac{k}{2}\left(R^{\prime} \quad R\right)
$$

$\mathrm{R}^{\prime} \rightarrow$ distância da borda do disco ao ponto de pressão no fluido;

$\mathrm{R} \rightarrow$ distância do centro do disco ao ponto de pressão no fluido; 
$\rho \rightarrow$ densidade do meio fluido;

$s \rightarrow$ velocidade do som no meio fluido, $(\lambda \sigma)$;

$\lambda \rightarrow$ comprimento de onda;

$v \rightarrow$ taxa de variação da amplitude da face do disco piezelétrico;

$\sigma \rightarrow$ frequência analisada do ultrassom;

$\mathrm{k} \rightarrow$ constante de propagação do som no meio, $(2 \pi \sigma / \mathrm{s})$.

Transdutores Magnetoestrictivos. O níquel, juntamente com outros metais ou ligas, variam de dimensão quando sujeitos a uma variação de campo magnético. Este efeito, conhecido como magnetoestricção, não é linear, como o efeito piezelétrico: para pequenas variações $\mathrm{dB}$ na densidade do fluxo magnético a partir de um valor inicial Bo, a tensão $S$ é proporcional a $B_{0} d B$. Se a densidade de fluxo é expressa em Weber $(\mathrm{Wb}=$ volt $\times \mathrm{S}=108$ linhas) por metro quadrado, o esforço é dado aproximadamente pela equação (2).

Onde:

$$
S=-0,002 B_{0} d B(2)
$$

Bo $\rightarrow$ é a função inicial do campo indutor aplicado ao material magnetoestrictivo;

$\mathrm{dB} \rightarrow$ variação infinitesimal do campo.

O movimento é caracterizado por uma onda senoidal em função do tempo, com expressões que dependem do deslocamento, da velocidade, da pressão, etc., com base no fator $e^{(j t)}$, onde $\omega$ é a frequência angular. Consequentemente, se $k$ for escrito como $\omega / \mathrm{s}$, a expressão para y assume a equação (3), onde A e B são constantes determinadas pelas condições de contorno.

$$
y=A e^{j(t k x)}+B e^{j(t+k x)}
$$

O decaimento da irradiação da potência acústica atua pela própria ação da expansão da frente de propagação, nesse caso, sob a forma de uma esfera que se expande, e pelo processo de contraposição e superposição das ações de compressão e cavitação acústicas, ou impedância acústica do meio de propagação, que, por sua vez, é função direta da interação entre viscosidade, densidade do meio e frequência da onda propagada.

A Equação (4) identifica as com o coeficiente de atenuação para a amplitude. É conveniente expressar o número de partículas $\mathrm{N}$ por unidade de volume em termos da razão $\square$ do volume ocupado pelo número total de partículas, dessa forma

$$
s=\frac{14^{4} a^{3}}{3^{4}}
$$

Com base nesse equacionamento foram projetados cenários de propagação de ondas ultrassônicas com base nos perfis de tanques que viabilizassem o projeto e construção do equipamento de desagregação ultrassônica. A primeira análise foi com base em um tanque de formato quadrado, projetando-se uma onda de reflexão de $2^{\underline{a}}$ ordem (primeira reflexão), conforme figura (1). 


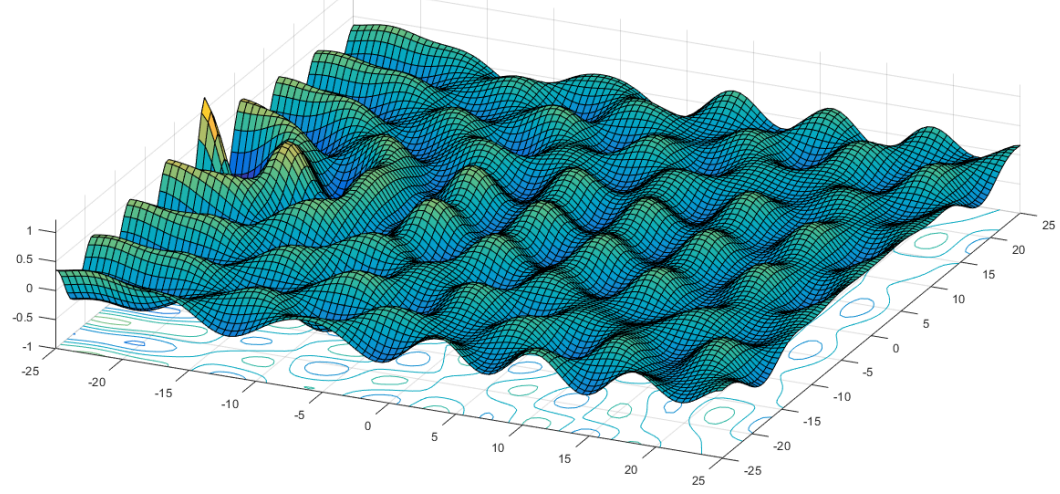

Fig. 1 - simulação tridimensional da propagação de ondas ultrassônicas de $16 \mathrm{kHz}$ em um tanque quadrado de $50 \mathrm{~cm}$ de aresta, com atenuação das ondas no meio aquoso, no MatLab.

Esta projeção leva em conta o efeito de reflexão das ondas acústicas nas paredes frontais e laterais do tanque, podendo ver o efeito das curvas isopotenciais de pressão acústica projetadas, conforme figura (2).

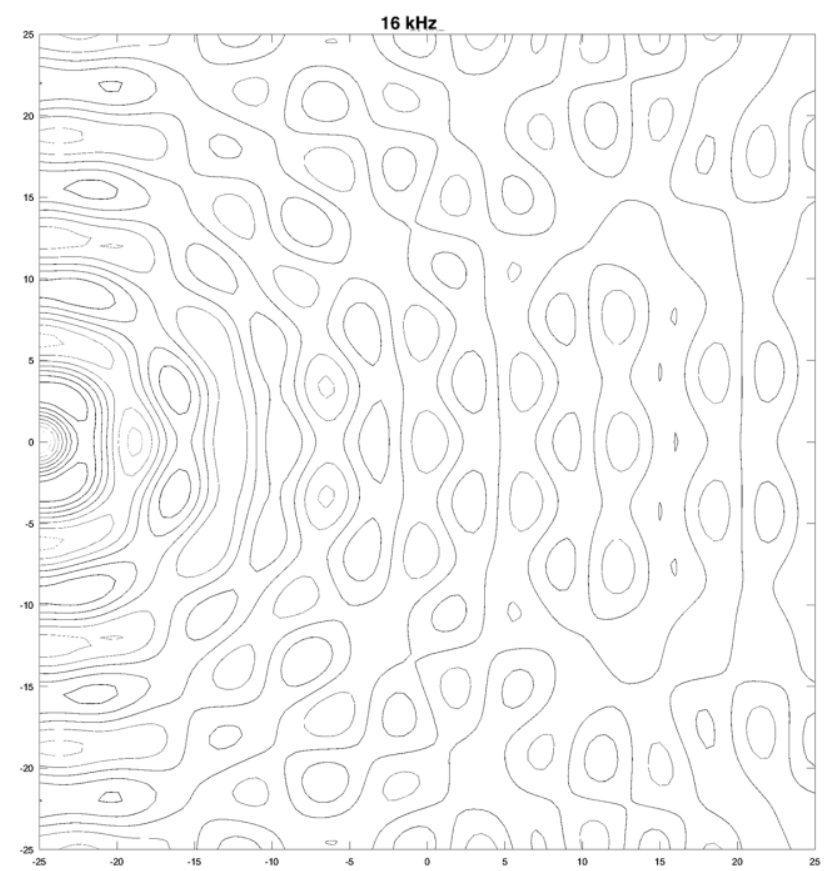

Figura 2. curvas isopotenciais de pressão acústica da frequência de $16 \mathrm{kHz}$ em um tanque quadrado de $50 \mathrm{~cm}$ de aresta, com atenuação das ondas no meio aquoso, no MatLab.

A comparação entre a secao de formato quadrado e circular mostra que a propagação de ondas e sua sobreposição de $2^{\mathrm{a}}$ ordem mostram que o tanque circular tem muito mais eficiência que o formato quadrado. Conforme figura 3. 


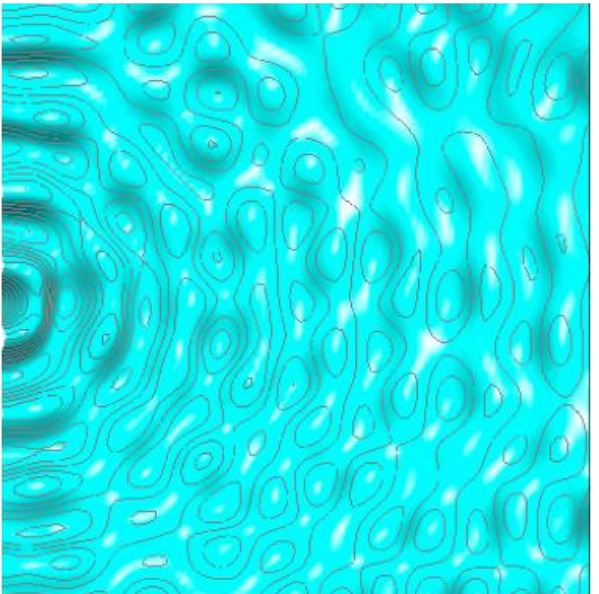

(a)

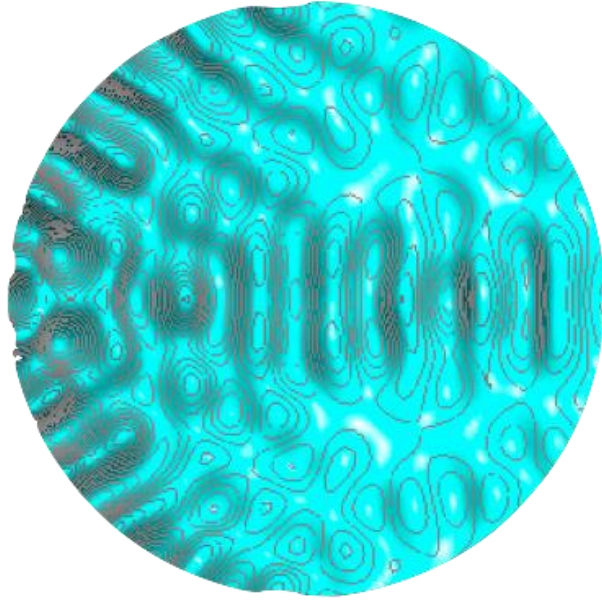

(b)

Figura 3. curvas isopotenciais de pressão acústica da frequência de $16 \mathrm{kHz}$, com atenuação das ondas no meio aquoso, em tanque: (a) de seção quadrada de $50 \mathrm{~cm}$ de aresta; (b) em tanque de seção circular com diâmetro de $50 \mathrm{~cm}$. Desenvolvidos no Grapher para Mac.

Em seguida desenvolveu-se o estudo referente à superposição contínua de ondas ortogonais, na tentativa de aumentar a eficiência de densidade de compressão e cavitação dentro do tanque. Conforme figura 4.

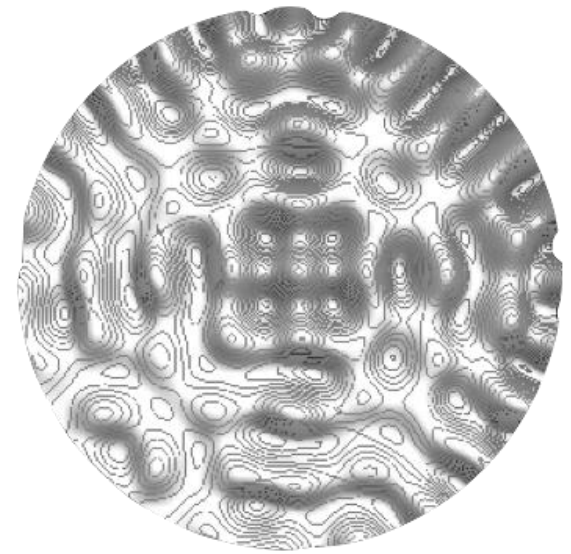

(a)

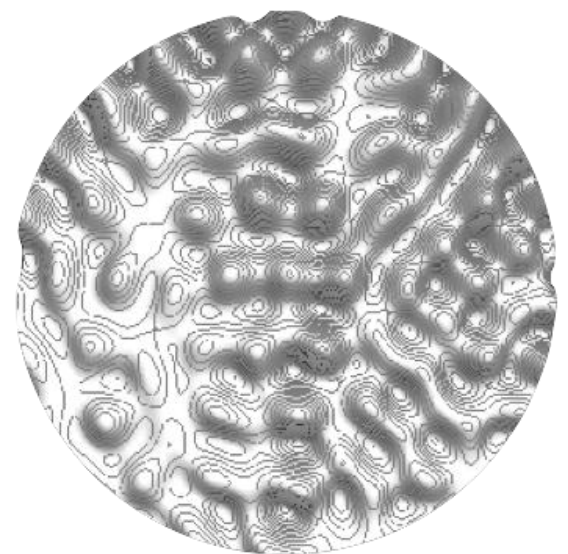

(b)

Figura 4. curvas isopotenciais de pressão acústica em tanque cilíndrico, com associação de ondas ortogonais entre si: (a) dupla sobreposição de ondas de $16 \mathrm{kHz}$; (b) dupla sobreposição de ondas de 16 e $20 \mathrm{kHz}$. Desenvolvidos no Grapher para Mac.

$\mathrm{Na}$ figura 4 podemos perceber que a falta se simetria entre as ondas de 16 e $20 \mathrm{kHz}$ criam um ambiente muito mais dinâmico para o tanque circular, tornando muito mais eficiente o efeito de dispersão do ultrassom nas lamas de aciaria.

A tabela 1 mostra os resultados médios das análises das lamas grossa e fina de aciaria LD utilizadas no estudo. A tabela 2 mostra o resultado médio da análise granuloquímica destas lamas.

O equipamento foi projetado inicialmente para ser capaz de assimilar uma vazão de 35 t/mês de produção, para finalmente, ser levado a uma capacidade de produção mensal de lama grossa de uma aciaria de grande porte, de cerca de 3.000 t/mês. 
Tabela 1 - Valores médios de composição para as frações grossa e fina da lama de aciaria, analisadas (SOUZA, 2006)

\begin{tabular}{|c|c|c|}
\hline Composto & Lama Grossa & Lama Fina \\
\hline Fe Total & $80,53 \%$ & $56,46 \%$ \\
\hline Fe - Metálico & $68,70 \%$ & $4,90 \%$ \\
\hline $\mathrm{Fe}_{2} \mathrm{O}_{3}$ & - & $5,25 \%$ \\
\hline $\mathrm{FeO}$ & $16,40 \%$ & $66,48 \%$ \\
\hline $\mathrm{SiO}_{2}$ & $3,65 \%$ & $4,25 \%$ \\
\hline $\mathrm{CaO}$ & $8,40 \%$ & $12,90 \%$ \\
\hline $\mathrm{Al}_{2} \mathrm{O}_{3}$ & $1,70 \%$ & - \\
\hline $\mathrm{MgO}$ & $0,50 \%$ & $5,60 \%$ \\
\hline $\mathrm{C}$ & $1,70 \%$ & - \\
\hline
\end{tabular}

Tabela 2 - Análise do teor de ferro metálico, $\mathrm{FeO}$ e $\mathrm{Fe}_{2} \mathrm{O}_{3}$, presentes na lama fina de aciaria, global e por faixa granulométrica (SOUZA, 2006)

\begin{tabular}{|c|c|c|c|c|c|c|}
\hline Granulometria & Massa (g) & Retida simples (\%) & $\mathrm{Fe}_{\text {total }}$ & $\mathrm{FeO}$ & $\mathrm{Fe}_{2} \mathrm{O}_{3}$ & $\mathrm{Fe}_{\mathrm{met}^{\left({ }^{*}\right)}}$ \\
\hline Global & 10.000 & $100,00 \%$ & $56,86 \%$ & $66,48 \%$ & $5,25 \%$ & $4,90 \%$ \\
\hline $65 \#$ & 4.887 & $35,51 \%$ & $56,41 \%$ & $66,50 \%$ & $5,96 \%$ & $3,81 \%$ \\
\hline $100 \#$ & 2.251 & $16,36 \%$ & $56,39 \%$ & $68,36 \%$ & $4,22 \%$ & $3,94 \%$ \\
\hline $150 \#$ & 418 & $30,37 \%$ & $58,22 \%$ & $64,78 \%$ & $5,07 \%$ & $5,80 \%$ \\
\hline 200\# & 836 & $6,07 \%$ & $59,51 \%$ & $68,87 \%$ & $3,11 \%$ & $5,96 \%$ \\
\hline $270 \#$ & 772 & $5,61 \%$ & $59,86 \%$ & $66,41 \%$ & $6,08 \%$ & $7,04 \%$ \\
\hline $325 \#$ & 322 & $2,34 \%$ & $61,10 \%$ & $68,76 \%$ & $6,60 \%$ & $5,63 \%$ \\
\hline $400 \#$ & 289 & $2,10 \%$ & $57,78 \%$ & $68,38 \%$ & $5,04 \%$ & $7,64 \%$ \\
\hline$<400 \#$ & 225 & $1,63 \%$ & $56,86 \%$ & $64,84 \%$ & $7,03 \%$ & $5,67 \%$ \\
\hline
\end{tabular}

\subsection{Cálculo da Relação de Potência}

Com base na relação potência e volume de polpa obtido nos testes de bancada, calculou-se uma relação de consumo e potencia para o tratamento de 1 tonelada de material.

- A Densidade medida do sólido por picnômetro ultrassonado: $d=5,35 \mathrm{~g} / \mathrm{cm}^{3}=5,35 \mathrm{~kg} / \mathrm{dm}^{3}$ $=5,35 \mathrm{~kg} / \mathrm{L}$.

- Para o tratamento ultrassônico em equipamento de bancada, $25 \mathrm{kHz}$, a polpa formada teve uma relação de massa sólido/polpa em torno de $25 \%$, sendo assim:

- Considerando uma potência específica $(\pi)$ ideal ao processo de 15 Watts/litro:

- Como o tempo de ultrassonagem durante a desagregação máxima, a partir do qual praticamente não ocorreram mudanças na eficiência do processo, foi de $\mathbf{3} \mathbf{~ m i n}$. Assim a energia gasta em uma tonelada de lama $(E)$, será:

A análise dos resultados levou à conclusão de que a lama grossa de aciaria sofre desagregação com uma relação de potência de cerca de $15 \mathrm{~W} / \mathrm{L}$ de polpa, com uma concentração que varia de 25 a $30 \%$ de sólidos na mesma. E estimou-se um consumo médio de 2,4 kWh/t, que foi confirmado na prática.

\subsection{Projeto e Construção de um Desagregador/Dispersor Ultrassônico}

Para a construção do equipamento para tratamento da lama de aciaria utilizou-se o resultado dos dados teóricos estimados, comprovados com os testes de laboratório e bancada. Com base nestes resultados foi projetado o tanque de dispersão ultrassônica.

O projeto construtivo dos transdutores utilizados foi feito pela Lewis Corporation, Co., onde o perfil de emissão ultrassônica consiste em uma fração da área frontal do corpo do equipamento transdutor, como pode ser visto na Figura 5. 

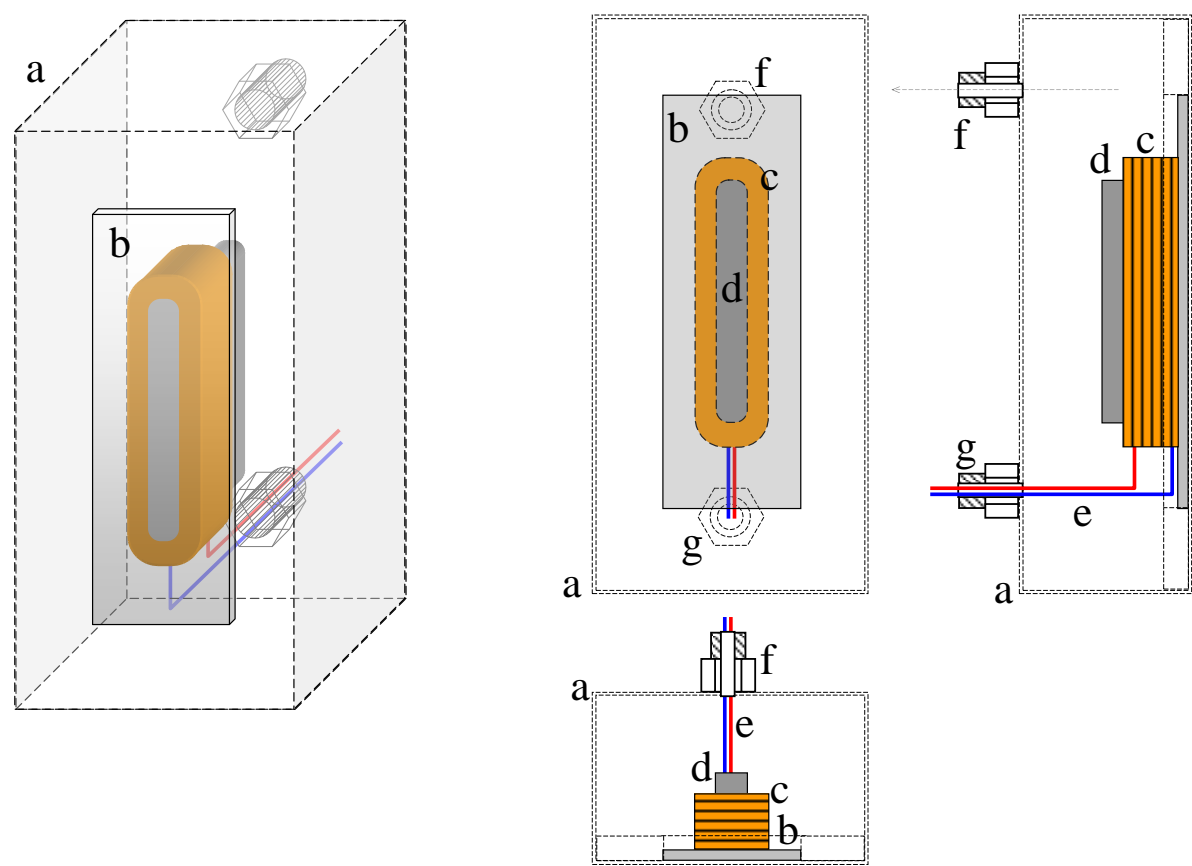

$\mathrm{a}$

Figura 5. Esquema construtivo do transdutor ultrassônico: (a) tanque de proteção do transdutor; (b) transdutor - placa magnetoestrictiva de níquel; (c) bobina do enrolamento de excitação magnética; (d) núcleo concentrador de alta permeabilidade magnética; (e) cabos de alimentação provenientes do gerador de ondas quadradas de frequência ultrassônica; (f) furo de saída de ar da câmara interna da caixa de proteção do ultrassom; (g) furo de entrada dos cabos de alimentação de pulsos elétricos.

Fonte: Souza (2006)

O primeiro projeto foi feito para uma vazão de polpa de aproximadamente $10 \mathrm{~L} / \mathrm{min}$, para uma polpa com concentração de sólido de cerca de $30 \%$, e um desagregador com uma potência de trabalho de $300 \mathrm{~W}$, com um tanque de cerca de $20 \mathrm{~L}$ de volume útil.

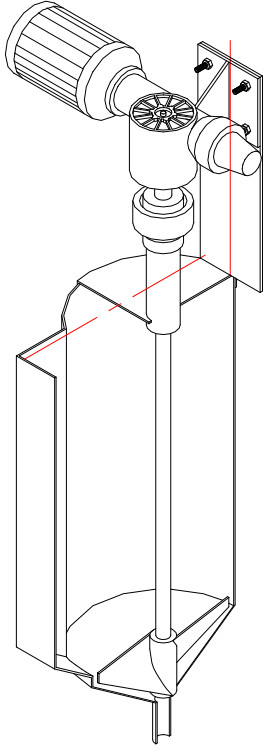

(a)

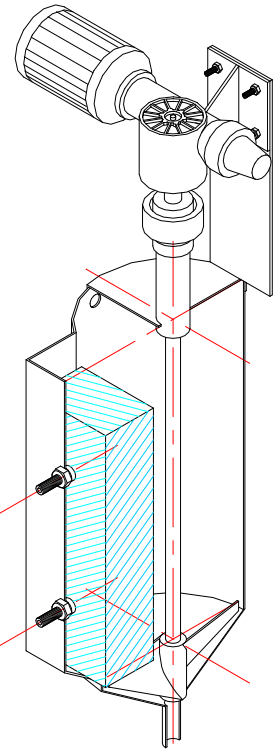

(b)

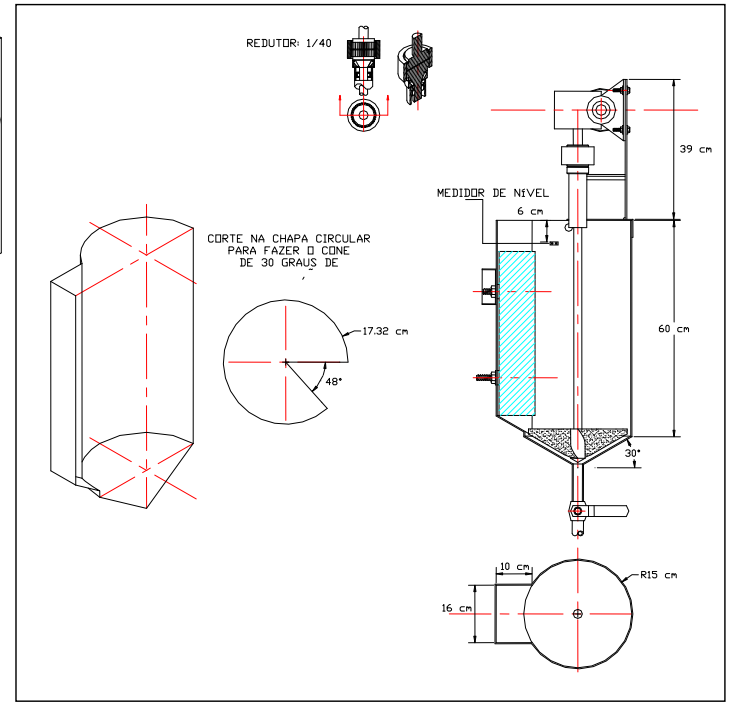

Figura 6. Projeto do tanque do protótipo de 300W: (a) tanque do agitador; (b) com o transdutor instalado. Fonte: Souza (2006) 


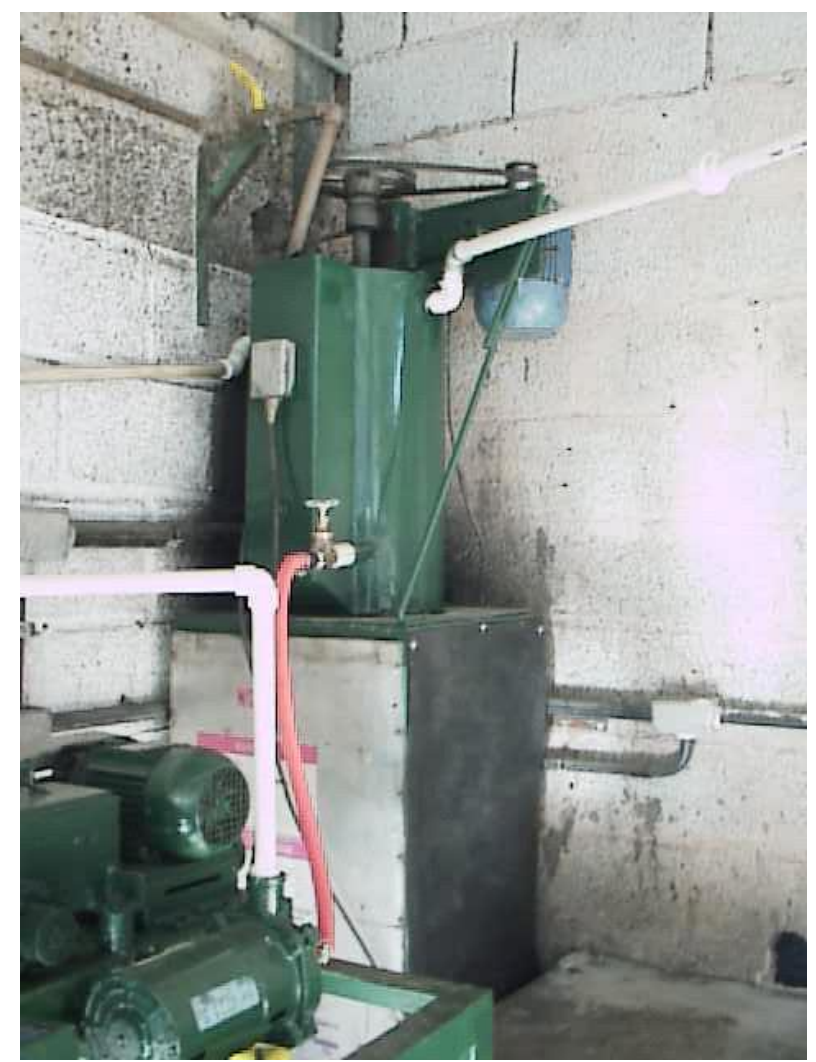

Figura 7. Protótipo do desagregador ultrassônico, com potência de $300 \mathrm{~W}$, construído a partir do projeto. Capacidade de 35 t/mês. (SOUZA, 2006)

\section{RESULTADOS E DISCUSSÃO}

\subsection{Análise dos testes na planta piloto}

O material tratado na planta piloto apresentou a seguinte distribuição de massa em relação ao seu teor metálico.

Tabela 3 - Distribuição de massa e teores metálicos (valores médios) obtidos para os componentes da lama grossa obtidos após processamento na planta piloto, 35 t/mês (SOUZA, 2006)

\begin{tabular}{lrrrr}
\hline \multirow{2}{*}{ Produto } & Massa $(\mathrm{g})$ & \multirow{2}{*}{ Fe Metálico $(\%)$} & \multicolumn{2}{c}{ Distribuição } \\
\cline { 4 - 5 } & $15.000,00$ & $72,03 \%$ & $100,00 \%$ & $100,00 \%$ \\
\hline Alimentação & $10.949,48$ & $95,21 \%$ & $73,00 \%$ & $96,49 \%$ \\
\hline Concentrado & $3.501,39$ & $10,65 \%$ & $23,34 \%$ & $3,45 \%$ \\
\hline Rejeito & 549,13 & $1,21 \%$ & $3,66 \%$ & $0,06 \%$ \\
\hline Finos (poeira) & & & &
\end{tabular}

Tabela 4 - Teores de recuperação de massa e teores metálicos da lama grossa de aciaria para a planta industrial, 1200 t/mês (SOUZA, 2006)

\begin{tabular}{lrrrr}
\hline \multirow{2}{*}{ Produto } & \multirow{2}{*}{ Massa $(\mathrm{kg})$} & Fe Metálico (\%) & \multicolumn{3}{c}{ Distribuição } \\
\cline { 4 - 5 } & $1.000,00$ & $72,15 \%$ & Massa $(\%)$ & \multicolumn{2}{c}{ Fe Metálico $(\%)$} \\
\hline Alimentação & 704,89 & $93,15 \%$ & $70,49 \%$ & $100,00 \%$ \\
\hline Concentrado & 221,28 & $27,71 \%$ & $22,13 \%$ & $91,01 \%$ \\
\hline Rejeito & 73,83 & $4,86 \%$ & $7,38 \%$ & $8,50 \%$ \\
\hline Finos (poeira) & & & &
\end{tabular}

A partir dos resultados obtidos pelas operações nas plantas piloto e da primeira planta industrial, tornou-se possível o projeto e construção de uma planta operacional a nível 
industrial em maior escala, preparada para tratar $3200 \mathrm{t} / \mathrm{mês}$, instalada e operada dentro da Companhia Siderúrgica Nacional, Usina Presidente Vargas, em Volta Redonda, RJ.

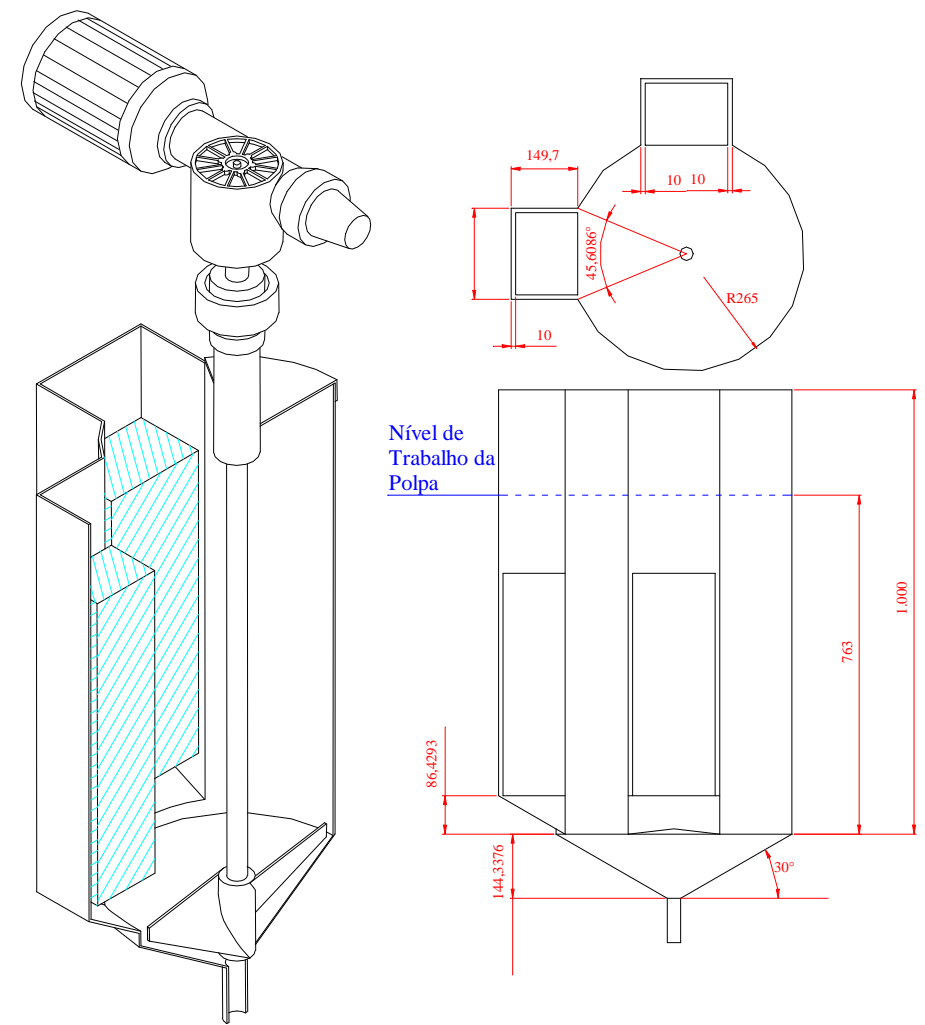

Figura 10. Projeto do tanque com dois transdutores ortogonais. Souza (2006)

Para a planta instalada na CSN, em Volta Redonda, RJ, utilizou-se um segundo transdutor ultrassônico, de frequência diferente do primeiro. Foram instalados dois transdutores, um de $16 \mathrm{kHz}$ e um de $20 \mathrm{kHz}$, ortogonalmente entre si, gerando um ambiente mais agitado e eficiente na desagregação. 

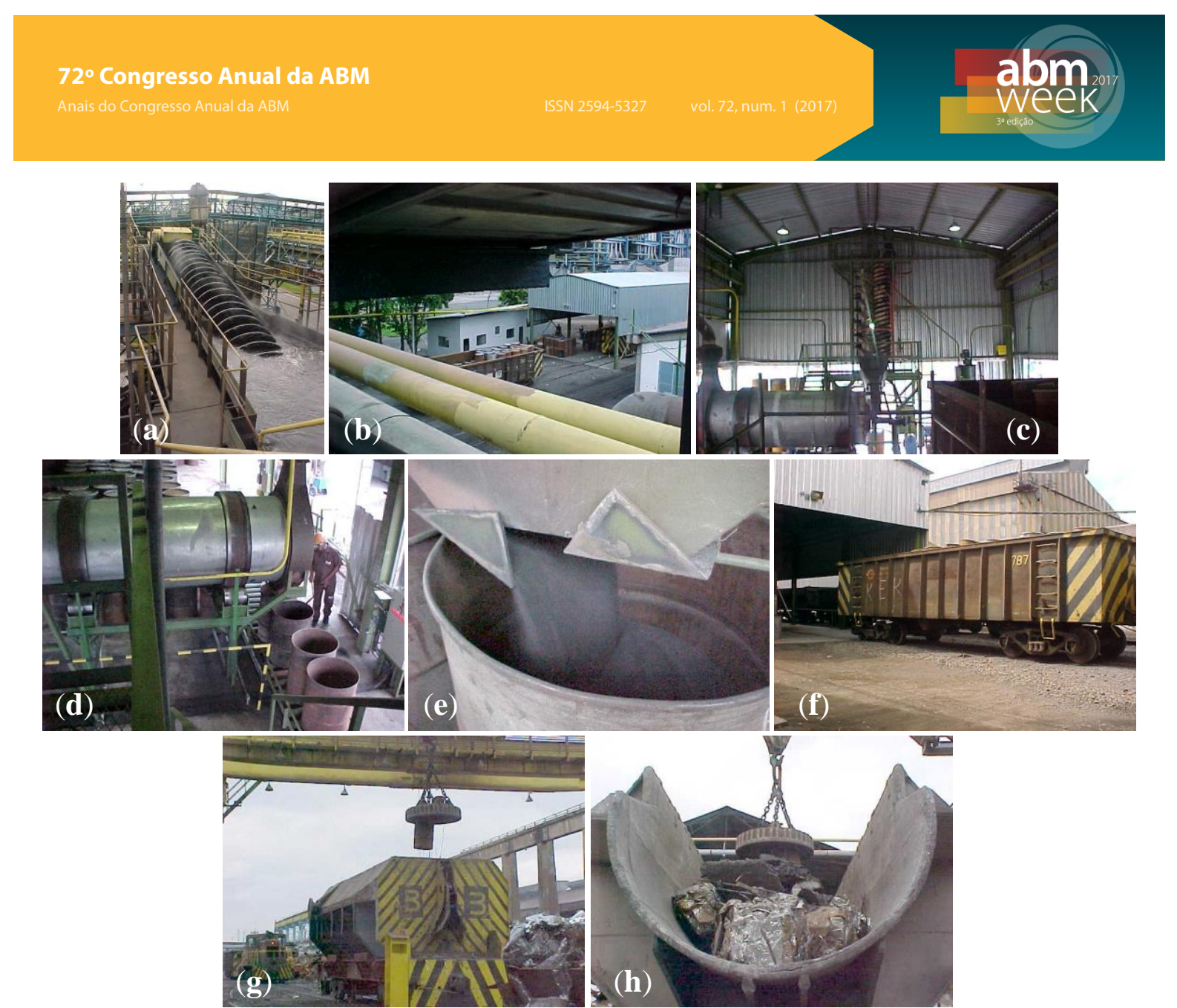

Figura 11. Planta Industrial, capacidade: 3200 t/mês, instalada na CSN (Volta Redonda, RJ - Brasil): (a) classificador parafuso retirando a lama grossa do espessador; (b) tubulação por onde a lama é bombeada para a planta de tratamento pelo D.U.; (c) planta de tratamento da lama de aciaria pelo processo D.U.; (d) forno de secagem onde o material é descarregado em tambores; (e) concentrado seco carregado em tambores; (f) vagão levando os tambores com o concentrado para alimentação na aciaria; (g) eletroímã carregando o tambor contendo o concentrado metálico na caixa de sucatas; (h) vista de frente da caixa de sucatas. Fonte: Souza (2006)

\subsection{Discussão dos Resultados}

O material tratado apresenta-se desagregado, livre dos colóides que o tornam uma lama uniforme. Após esta dispersão o material é tratado em espirais de Reichert, para sua concentração gravítica. A figura 12 mostra este resultados de concentração. Cada tonelada de esfera recuperada é capaz de evitar 1,4 tonelada de minério a ser consumido pelo altoforno, $440 \mathrm{~kg}$ de coque a ser queimado e 1,5 toneladas de $\mathrm{CO}_{2}$ a serem geradas. $\mathrm{O}$ cálculo é simples:

1 tonelada de gusa (94\% $\mathrm{Fe}$ ): $940 \mathrm{~kg}$ de $\mathrm{Fe} \rightarrow 1000 \mathrm{~kg}$ gusa $\rightarrow 1400 \mathrm{~kg}$ minério 1 tonelada de concentrado (93,15\% Fe): $931,5 \mathrm{~kg}$ de $\mathrm{Fe} \rightarrow 991 \mathrm{~kg}$ gusa $\rightarrow 1387 \mathrm{~kg}$ minério Cada tonelada de gusa consome:

$484 \mathrm{~kg}$ Coque $(84 \%$ C) - $991 \mathrm{~kg}$ de Gusa $\rightarrow 402,8 \mathrm{~kg} \mathrm{C}$

$1 \mathrm{~kg}$ de $\mathrm{C} \rightarrow 3,67 \mathrm{~kg} \mathrm{CO} 2$

$479,6 \mathrm{~kg}$ Coque $\rightarrow 402,8 \mathrm{~kg} \mathrm{C} \rightarrow 1477,2 \mathrm{~kg} \mathrm{CO}_{2}$ 

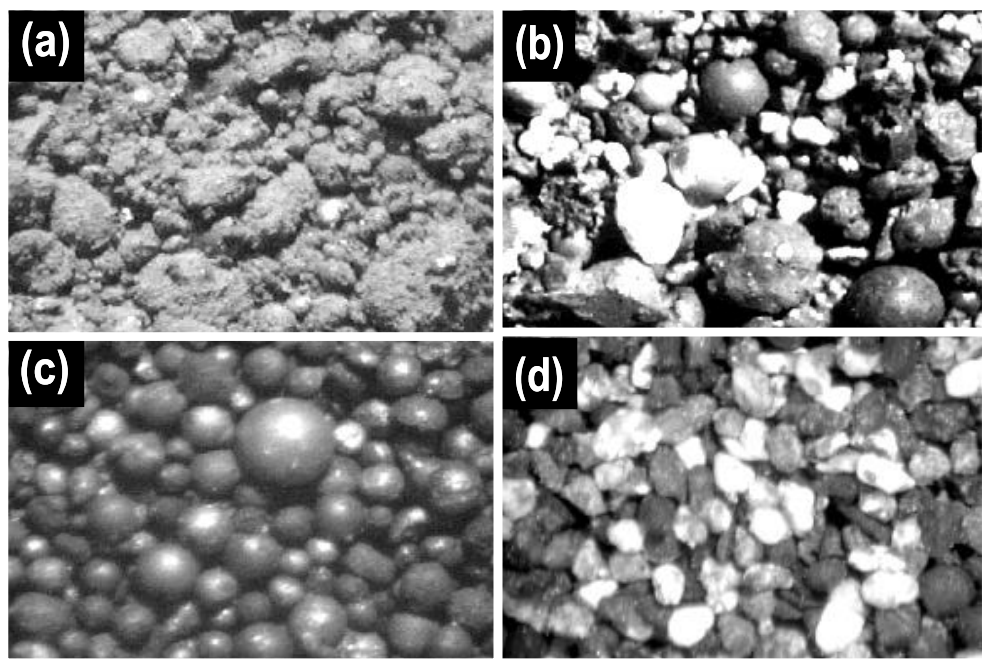

Figura 12. Aspecto da lama grossa nas várias etapas correspondentes ao processo: (a) lama grossa original seca; (b) após desagregação pelo D.U., com retirada dos finos coloidais; (c) concentrado metálico, rico em ferro, sob a forma de microesferas; (d) resíduo secundário do processo, rico em $\mathrm{CaO}$, gerado pelas espirais de Reichert. Fonte: Souza (2006)

\section{CONCLUSÃo}

- O D.U. (Desagregador Ultrassônico) tem uma eficiência significativa pois seu consumo de energia em uma planta industrial (referência à planta instalada na CSN, em Volta Redonda, RJ) não ultrapassa, em todo o processo, 3kWh/t lama;

- O aspecto ambiental é notavelmente preservado pois, o processo inteiro decorre por ação unicamente física, sem qualquer tipo de contaminação química tanto da polpa quanto da água utilizada, permitindo que essa água, após decantação de seus particulados suspensos, retorne ao processo;

- A recuperação e reciclagem deste teor metálico como sucata faz com que, para cada tonelada de material reciclado, deixe-se de consumir 1,4 toneladas de minério, 484 $\mathrm{kg}$ de Coque e deixe-se de produzir 1,5 toneladas de $\mathrm{CO}_{2}$, para a mesma produção de aço;

- O D.U. também atua significativamente na recuperação da lama fina de aciaria, viabilizando sua reciclagem em processos de sinterização e em processos fora da indústria, tais como fabricação de cerâmica, etc.;

- Em função de um custo razoavelmente baixo de instalação e da simplicidade da operação constitui-se em um processo que viabiliza-se técnica e economicamente para instalação e operação.

\section{Agradecimentos}

Agradeço ao CNPq, CAPES, Fundação Gorceix, DTECH/UFSJ, ao Engenheiro Rogério Veiga e a todos que contribuíram para o sucesso deste projeto.

\section{REFERÊNCIAS}

1 VIGOUREUX, P. "Ultra-Sonics”. Royal Naval Scientific Service. Ed. Chapman and Hall, 1942.

2 SOUZA, E. L. Estudo e Desenvolvimento de um Processo de Tratamento de Resíduos Siderúrgicos Através da Técnica de Bombardeamento Ultra-Sônico. Tese de Doutorado. REDEMAT/UFOP. 2006. 
3 SOUZA, E. L.; ARAÚJO, F.G.S.; OLIVEIRA, C.P.; GOMES, J. E. L. Improvement of Metallic Joint Electrical Conductivity Using a Novel Conductive Paste Produced from Recycled Residues. Revista Escola de Minas. 2006, Vol. 59.

4 Wozniacki, Z.; Telejko, T.; Kenig R. Sintering as the method of utilization of steelmaking dusts with a high content of zinc oxides. Hutnik-WH 81, 166-171 (2014).

5 Steel Dust Recycling, Waelz Kiln Technology. Available: http://www.globalsteeldust. com/waelz kiln technology [06/10 2016].

6 Gandiaga, N.G.; Vergara, B.G.E. Proceedings of the "Rewas" 99: Global symposium on recycling, waste treatment and clean technology, TMS, INASMET, San Sebastian, (1999) pp. 1511-152. 